\title{
NUMERICAL SHADOWING USING COMPONENTWISE BOUNDS AND A SHARPER FIXED POINT RESULT*
}

\author{
ERIK S. VAN VLECK ${ }^{\dagger}$
}

\begin{abstract}
Shadowing provides a means of obtaining global error bounds for approximate solutions of nonlinear differential equations with interesting dynamics, in particular, for initial value problems with positive Lyapunov exponents. Shadowing breaks down in the presence of zero Lyapunov exponents, although some results such as shadowing with rescaling of time have been obtained. Using a reformulation of the original differential equations and an improved fixed point result we take advantage of componentwise local error bounds to use relatively smaller error tolerances in nonhyperbolic and contractive directions (i.e., in directions corresponding to zero and negative Lyapunov exponents). The result is a decrease in the shadowing global error.
\end{abstract}

Key words. shadowing lemma, hyperbolicity, global error estimation

AMS subject classification. 65L

PII. S1064827599353452

1. Introduction. Global error analysis using a shadowing lemma approach involves treating the error equation as a boundary value problem (BVP) as opposed to an initial value problem (IVP). This allows for shadowing-type error analysis results for problems with expansive and contractive directions. The difficulty occurs when there are directions that are neither exponentially expansive nor contractive, and we call these nonhyperbolic directions. Most shadowing lemma techniques rely on a fixed point result that is similar to using the numerically computed solution as an initial guess for a modified Newton's method to find a true trajectory to the given differential equation. This leads to the need to find and bound an inverse (in our case a right inverse) of the Jacobian for the modified Newton's method.

In this paper, we consider an improved fixed point result and find that what is important is not so much the norm of a right inverse of the Jacobian, but the action of the right inverse on several vectors. This produces sharper results and the ability to gain a better understanding of the breakdown of shadowing in the presence of nonhyperbolic directions. We reformulate the original differential equation in terms of a new coordinate system that corresponds to the directions associated with the Lyapunov exponents of the linearized problem that we denote by the columns of an orthogonal matrix function $Q \equiv Q(t)$. This allows us to write the dependent variable $x$ of the nonlinear differential equation in terms of these directions of different growth and decay rates and write $x=Q y$ and solve for $Q$ and $y$ instead of $x$. With this reformulation we derive componentwise bounds on the magnitude of the local error in directions corresponding to different rates of growth and decay, and with the improved fixed point result we are able to derive sharper shadowing bounds by employing smaller local error tolerances in the nonhyperbolic and contractive directions.

Our focus numerically is in employing existing IVP solvers, including the extrapolation code ODEX from [17] and the Runge-Kutta suite RKSUITE from [3]. The

\footnotetext{
* Received by the editors March 22, 1999; accepted for publication (in revised form) February 21, 2000; published electronically August 31, 2000.

http://www.siam.org/journals/sisc/22-3/35345.html

${ }^{\dagger}$ Department of Mathematical and Computer Sciences, Colorado School of Mines, Golden, CO 80401-1887 (evanvlec@mines.edu). The work of this author was supported by NSF grants DMS9505049 and DMS-9973393.
} 
numerical shadowing methods presented here provide accurate results, due to the componentwise error bounds and the improved fixed point result, and are obtained with relatively small cost due to the use of efficient numerical linear algebra techniques.

Recent work on shadowing has involved extending shadowing to allow for rescaling of time, i.e., allowing for perturbations in the time step (see the work of Coomes [6], Coomes, Kocak, and Palmer [7], Palmer [22], Van Vleck [26], and Franke and Selgrade [12], [13]). Rescaling of time can be thought of as allowing for a special perturbation of the differential equation. Work on numerical shadowing includes the ground breaking work of Hammel, Yorke, and Grebogi [18], [19], the shadowing-type results of Beyn [2], the work of Chow, Lin, and Palmer (e.g., [4]), Chow and Palmer (e.g., [5]), the numerical work of Sauer and Yorke [24], and the initial work on the breakdown of shadowing of Dawson et al. [8]. We also mention here a related concept known as backward error analysis in which one considers a modified differential equation and provides error statements with respect to this modified equation. This has proved to be a very useful concept of error, especially in the case of Hamiltonian systems (see, e.g., [1], [15], [16], and [23]).

This paper is organized as follows. In section 2 we present the shadowing setup that we will employ throughout this paper, and in section 3 we present an improved fixed point result that has two fewer norm bounds than the previous result. In section 4 we consider a reformulation of the given differential equation and its linear variational equation by writing the dependent variable $x(t)$ as $x(t)=Q(t) y(t)$ and a fundamental matrix solution $X(t)=Q(t) R(t)$, respectively, where $Q(t)$ is a square orthogonal matrix and $R(t)$ is an upper triangular matrix, and by solving for $y, Q$, and $R$ instead of $x$ and $X$. Based upon this reformulation, we consider in section 5 the form of a right inverse, in our case the pseudoinverse, of a linear operator that approximates the Jacobian, and we provide bounds in section 6 on the elements of the vectors multiplying the right inverse. Section 7 contains algorithmic formulations and details, section 8 contains our numerical results, and section 9 contains our conclusions.

2. Shadowing setup. Consider the autonomous IVP where $f$ is a $C^{2}$ function,

$$
\left\{\begin{aligned}
\dot{x} & =f(x) \\
x(0) & =x_{0}
\end{aligned}\right.
$$

and $x \equiv x(t): \mathbb{R} \rightarrow \mathbb{R}^{N}$ for a positive integer $N$.

We assume that a numerical orbit $x \equiv\left\{x_{n}\right\}_{0}^{M}$ using time steps $h \equiv\left\{h_{n}\right\}_{0}^{M-1}$, for a positive integer $M$, has been produced, and let $t_{n+1}=t_{n}+h_{n}$ with $t_{0}=0$. Consider the nonlinear operator $F(x, h)$ with $n$th block component given by

$$
(F(x, h))_{n}=x_{n+1}-\phi\left(x_{n}, h_{n}\right)=: \delta_{n+1},
$$

for $n=0, \ldots, M-1$, where $\phi$ is the local solution operator and $\delta_{n+1}$ is the local error at the $n$th step. The linearization of $F(x, h)$ is given by $\left(D_{x_{n}} \phi\left(x_{n}, h_{n}\right) \equiv \frac{\partial \phi}{\partial x_{n}}\left(x_{n}, h_{n}\right)\right.$ and $\left.D_{h_{n}} \phi\left(x_{n}, h_{n}\right) \equiv \frac{\partial \phi}{\partial h_{n}}\left(x_{n}, h_{n}\right)\right)$,

$$
(D F(x, h)(\Delta x, \Delta h))_{n}=\Delta x_{n+1}-D_{x_{n}} \phi\left(x_{n}, h_{n}\right) \Delta x_{n}-\theta D_{h_{n}} \phi\left(x_{n}, h_{n}\right) \Delta h_{n},
$$

for $n=0, \ldots, M-1$, where $\theta \geq 0$ is a scaling factor (see [26]). We assume that $D F(x, h)$ is approximated by a linear operator,

$$
(L(\Delta x, \Delta h))_{n}=\Delta x_{n+1}-Y_{n} \Delta x_{n}-\theta f_{n+1} \Delta h_{n},
$$


for $n=0, \ldots, M-1$. Throughout the paper we consider $z=x \in \mathbb{R}^{(M+1) N}$ for $\theta=0$ and $z=(x, h) \in \mathbb{R}^{(M+1) N} \times \mathbb{R}^{M}$ for $\theta>0$, with norms $\|z\|=\sup _{n}\left\|x_{n}\right\|$ for $\theta=0$ and $\|z\|=\|(x, h)\|=\max \left\{\sup _{n}\left\|x_{n}\right\|, \sup _{n}\left|\theta^{-1} h_{n}\right|\right\}$ for $\theta>0$. For $n=0, \ldots, M-1$ the $n$th $N \times N$ block column of $D F(z)$ and of $L$ is nonzero only in the $n$th block component. In addition, for $\theta>0$ the remaining $M$ columns of $D F(z)-L$ contain a nonzero block component of the form $\theta\left[f_{n+1}-D_{h_{n}} \phi\left(x_{n}, h_{n}\right)\right]$. The $n$th block component of $F(z)$ is given by $\delta_{n+1}$.

3. Fixed point result. The following is an improved version of a fixed point result that has been used in many instances to prove the existence of a nearby solution in a shadowing context. The proof is basically the same, but it allows for the use of more precise componentwise information. The statement of the fixed point theorem is very similar to the statement of Theorem 1, p. 536, in Kantorovich and Akilov [20], and the proof is a minor modification of Proposition 4.1 in [4].

TheOREm 3.1. Suppose that $\mathcal{X}, \mathcal{Y}$ are Banach spaces, $F: \mathcal{X} \rightarrow \mathcal{Y}$ is $C^{1}$, and that there exist positive constants $\epsilon_{0}, \alpha<1, \beta$, a point $z \in \mathcal{X}$, and a linear operator $L: \mathcal{X} \rightarrow \mathcal{Y}$ with right inverse $L^{\dagger}$ such that

$$
\left\|L^{\dagger}(D F(w)-L)\right\| \leq \alpha \quad \text { for } \quad\|w-z\| \leq \epsilon_{0} .
$$

If, for $0<\epsilon \leq \epsilon_{0}$,

$$
\left\|L^{\dagger} F(z)\right\| \leq(1-\alpha) \epsilon=: \beta,
$$

then the equation $F(w)=0$ has a solution with

$$
\|w-z\| \leq \epsilon
$$

Our goal now is to find a bound $\alpha<1$ in (3.1) and a bound on $\beta$ in (3.2) so that we can set $\epsilon=\beta /(1-\alpha)$ provided $\epsilon \leq \epsilon_{0}$.

4. Reformulation of the differential equation. In order to decrease the shadowing distance when there are nonhyperbolic directions, for instance, in the neighborhood of a nontrivial attracting set or in the presence of first integrals, we rewrite the differential equation (2.1) to take advantage of componentwise local error tolerances. We introduce an auxiliary matrix differential equation that will also be useful in solving the linear variational equation. To make the idea clear, suppose for each time $t$ we have an orthogonal matrix $Q(t) \in \mathbb{R}^{N \times N}$ whose columns correspond to directions of different rates of growth. Then we can write $x(t)$, the solution to $(2.1)$, as a linear combination of the columns of $Q(t)$, i.e., $x(t)=Q(t) y(t)$. The components of $y(t)$ correspond to the growth rates in the directions given by the columns of $Q(t)$. If we write the solution to the linear variational equation, $X(t)$, as $X(t)=Q(t) R(t)$ (see [9]), where $Q(t)$ is orthogonal and $R(t)$ is upper triangular, then $Q(t)$ satisfies the following matrix differential equation, where $S(Q) \equiv S(Q, A(t))$ is a skew-symmetric matrix and $A(t) \equiv D f(x(t))$,

$$
\dot{Q}=Q S(Q) \quad \text { with } \quad S(Q)_{i j}=\left\{\begin{array}{rr}
\left(Q^{T} A Q\right)_{i j}, & i>j, \\
0, & i=j \\
-\left(Q^{T} A Q\right)_{j i}, & i<j .
\end{array}\right.
$$

Since $x(t)=Q(t) y(t)$, we have $\dot{x}(t)=\dot{Q}(t) y(t)+Q(t) \dot{y}(t)$, so $y(t)$ satisfies the differential equation

$$
\dot{y}=Q^{T} f(Q y)-S(Q) y .
$$


The equation for $R(t)$ is given by

$$
\dot{R}=\tilde{A} R \quad \text { with } \quad \tilde{A}=Q^{T} A Q-S(Q) ;
$$

the coefficient matrix $\tilde{A}(t)$ is thus upper triangular.

By solving numerically (4.1), (4.2), and (4.3), we obtain approximations to the original nonlinear problem and the linear variational equation. If we write $x_{n+1}=$ $Q_{n+1} y_{n+1}$ and $\phi\left(x_{n}, h_{n}\right)=\tilde{Q}_{n+1} \psi\left(y_{n}, h_{n}\right)$, where $\psi$ is the local solution operator for (4.2), then by (2.2), we have

$$
Q_{n+1}^{T} \delta_{n+1}=Q_{n+1}^{T}\left[x_{n+1}-\phi\left(x_{n}, h_{n}\right)\right]=y_{n+1}-\psi\left(y_{n}, h_{n}\right)+\left(\tilde{Q}_{n+1}^{T}-Q_{n+1}^{T}\right) \phi\left(x_{n}, h_{n}\right) .
$$

We solve using (4.2) as opposed to (2.1) so that componentwise local error control may be obtained with respect to the moving coordinate system given by $Q(t)$. This could be accomplished by solving with (2.1) but would require that the error control for the $x$ variable depend on our approximation to $Q$. Solving by using (4.1) and (4.3) is a numerically stable way of approximating the linear variational equation.

5. Form of the pseudoinverse. We choose for the right inverse, $L^{\dagger}$, in Theorem 3.1, the pseudoinverse. This allows us to find $L^{\dagger}$ explicitly, which is useful when determining the bounds for $\alpha$ and $\beta$ in (3.1) and (3.2). The choice of the pseudoinverse also has the advantage that it is independent of the dynamics of the problem, i.e., does not depend on the number of stable, unstable, or neutral modes, and it is the optimal choice with respect to the two norm.

We solve (4.1) and (4.2) numerically to produce sequences $\left\{Q_{n}\right\}_{0}^{M}$ and $\left\{y_{n}\right\}_{0}^{M}$. Simultaneously, for $n=0, \ldots, M-1$, we solve

$$
\left\{\begin{array}{l}
\dot{\tilde{R}}_{n}(t)=\tilde{A}\left(t+t_{n}\right) \tilde{R}_{n}(t), \\
\tilde{R}_{n}(0)=I
\end{array}\right.
$$

for $0 \leq t \leq h_{n}$. If we let $R_{n}$ denote the approximation to $\tilde{R}\left(h_{n}\right)$, then in (2.4) we have $Y_{n}=Q_{n+1} R_{n} Q_{n}^{T}$. Notice that $L$ in (2.4) can be written as $L=\bar{Q} U \hat{Q}^{T}$, where $\bar{Q}, \hat{Q}$ are block diagonal matrices given by $\bar{Q}=\operatorname{diag}\left(Q_{1}, \ldots, Q_{M}\right), \hat{Q}=\operatorname{diag}\left(Q_{0}, \ldots, Q_{M}, I_{M}\right)$, where $I_{M}$ is the $M \times M$ identity matrix, and in block indices,

$$
(U(z, \tau))_{n}=z_{n+1}-R_{n} z_{n}-\theta g_{n+1} \tau_{n}
$$

where $f_{n+1}=Q_{n+1} g_{n+1}$ and $f_{n+1}=f\left(x_{n+1}\right)$. Thus, the pseudoinverse of $L, L^{\dagger}$, can be written as

$$
L^{\dagger}=\hat{Q} U^{\dagger} \bar{Q}^{T}
$$

where $U^{\dagger}$ is the pseudoinverse of $U$. We write $U^{\dagger}=U^{T}\left(U U^{T}\right)^{-1}$ and note that $U U^{T}$ is symmetric and block tridiagonal with entries $\left(U U^{T}\right)_{n, n}=I+R_{n} R_{n}^{T}+\theta^{2} g_{n+1} g_{n+1}^{T}$ for $n=0, \ldots, M-1$ and $\left(U U^{T}\right)_{n-1, n}=-R_{n}^{T}$ for $n=1, \ldots, M-1$.

We decompose $L^{\dagger}$ as in (5.3) and determine componentwise bounds on $\bar{Q}^{T}[D F(w)$ $-L]$ and $\bar{Q}^{T} F(z)$. Once componentwise bounds are obtained we find the bounds $\alpha$ and $\beta$ in (3.1) and (3.2), respectively, using the following simple idea. Let $u$ denote 
an arbitrary column of $\bar{Q}^{T}[D F(w)-L]$ or let $u$ denote $\bar{Q}^{T} F(z)$. Assume that the $i$ th component of $u, u_{i}$, satisfies $\left|u_{i}\right| \leq \gamma_{i}$ for some $\gamma_{i}>0$. If $y=\hat{Q} U^{\dagger} u$, then we have

$$
\left|y_{i}\right| \leq \sum_{j}\left|\left(\hat{Q} U^{\dagger}\right)_{i j}\right| \cdot \gamma_{j}
$$

Using the results in [21] and the references therein we have that the $(i, j)$ block of $\left(U U^{T}\right)^{-1}$ has the form

$$
\Gamma_{i j} \equiv\left(U U^{T}\right)_{i j}^{-1}= \begin{cases}\Psi_{i} \Omega_{j}^{T} & \text { for } i \leq j \\ \Omega_{i} \Psi_{j}^{T} & \text { for } i>j\end{cases}
$$

where $\Psi_{i}$ and $\Omega_{j}$ are $N \times N$ matrices. In addition, the block vectors $\Psi=\left(\Psi_{0}^{T}, \ldots, \Psi_{M-1}^{T}\right)^{T}$ and $\Omega=\left(\Omega_{0}^{T}, \ldots, \Omega_{M-1}^{T}\right)^{T}$ can be determined by solving

$$
\left(U U^{T}\right) \Omega=\Xi_{0} \quad \text { and } \quad\left(U U^{T}\right) \Psi=\Xi_{M-1} \Omega_{M-1}^{-T},
$$

where $\Xi_{0}$ denotes the block vector with the identity matrix in the 0th block and $\Xi_{M-1}$ denotes the block vector with the identity in the $(M-1)$ th block (with all other blocks zero). In general, (5.6) is not a numerically stable way to form $\left(U U^{T}\right)^{-1}$.

In the next section we will obtain componentwise bounds on $\bar{Q}^{T} F(z)$ and $\bar{Q}^{T}[D F(w)$ $-L]$ to form the vectors that will multiply the matrix $\hat{Q} U^{\dagger}$ which has, using the form of $\left(U U^{T}\right)^{-1}$, the block entries,

$$
\left(\hat{Q} U^{\dagger}\right)_{i j}=\left\{\begin{aligned}
-Q_{0} R_{0}^{T} \Gamma_{0, j}, & i=0, \\
Q_{i}\left[\Gamma_{i-1, j}-R_{i}^{T} \Gamma_{i, j}\right], & i=1, \ldots, M-1, \\
Q_{M} \Gamma_{M-1, j}, & i=M
\end{aligned}\right.
$$

for $j=0, \ldots, M-1$, and for $\theta>0$, the remaining $M$ rows of $\hat{Q} U^{T}\left(U U^{T}\right)^{-1}$ have the form

$$
-\theta\left(g_{i}^{T} \Gamma_{i-1,0}, \ldots, g_{i}^{T} \Gamma_{i-1, M-1}\right) \quad \text { for } \quad i=1, \ldots, M .
$$

Note that, by using the form of $L$, we have (for $\theta=0$ ) that $(2.2)$ is of the form

$$
Q_{n+1}^{T} \Delta x_{n+1}-R_{n} Q_{n}^{T} \Delta x_{n}=Q_{n+1}^{T} \delta_{n+1},
$$

which after a change of variables $\left(z_{n}=Q_{n}^{T} \Delta x_{n}\right.$ and $\left.\rho_{n}=Q_{n}^{T} \delta_{n}\right)$ has the form

$$
z_{n+1}-R_{n} z_{n}=\rho_{n+1}
$$

Consider the following simplified example of (5.10) with

$$
R_{n}=\left(\begin{array}{ccc}
R_{11} & R_{12} & R_{13} \\
0 & R_{22} & R_{23} \\
0 & 0 & R_{33}
\end{array}\right)
$$

where $R_{11}>1,0<R_{33}<1, R_{22}=1$, and $\rho_{n} \equiv \rho$ for all $n$. If we solve (5.10) forward in time for the second and third components of $z$ with initial conditions of 0 and backward in the first component of $z$ with terminal condition 0 , then we obtain linear growth in the solution $z$ unless we set the second and third components of $\rho$ to $O\left(M^{-1}\right)$ times the first component of $\rho$. This suggests having, after considering 
(4.4), a relatively smaller local error tolerance for the components of $y$ and columns of $Q$ that correspond to nonhyperbolic and contractive directions (assuming that the directions are ordered as in our simple example). Similarly, we will use a relatively smaller local error tolerance for the rows of $R$ corresponding to nonhyperbolic and contractive directions. It is important to point out that, although our plan is to require smaller error tolerances when computing the coupled set of equations for $y$, $Q$, and $R$ numerically, this should really only restrict our time step in the integration of $Q$ since the time step for $y$ and $R$ should be based on the unstable directions.

6. Bounds on $\overline{\boldsymbol{Q}}^{\boldsymbol{T}} \boldsymbol{F}(z)$ and $\overline{\boldsymbol{Q}}^{\boldsymbol{T}}[\boldsymbol{D F}(\boldsymbol{w})-\boldsymbol{L}]$. In what follows, let U denote the set of indices corresponding to unstable directions and let CS denote the set indices corresponding to nonhyperbolic and stable directions based upon some criteria, for instance, the average magnitude of the diagonal elements of the upper triangular coefficient matrix $\tilde{A}(t)$ from (4.3).

We first consider the case in which $\theta=0$ and wish to determine componentwise bounds on $Q_{n+1}^{T} \delta_{n+1}$ and on $Q_{n+1}^{T}\left[D_{w_{n}} \phi\left(w_{n}, h_{n}\right)-Y_{n}\right]$, which we rewrite as

$$
\begin{aligned}
& Q_{n+1}^{T}(D F(w)-L)_{n, n} \\
& \quad:=Q_{n+1}^{T}\left[D_{w_{n}} \phi\left(w_{n}, h_{n}\right)-D_{x_{n}} \phi\left(x_{n}, h_{n}\right)\right]+Q_{n+1}^{T}\left[D_{x_{n}} \phi\left(x_{n}, h_{n}\right)-Y_{n}\right] .
\end{aligned}
$$

For $0 \leq t \leq h_{n}$ define $y_{n}(t)$ and $Y_{n}(t)$ with $y_{n}(0)=x_{n}$ and $Y_{n}(0)=I$ as the numerical solution to the original nonlinear problem and the linear variational equation, respectively, using dense or continuous output (see [17, p. 176]). Let $E_{n}(t):=$ $D_{x_{n}} \phi\left(x_{n}, t\right)-Y_{n}(t)$ for $0 \leq t \leq h_{n}$, where $E_{n}(0)=0$. In the following lemma we consider componentwise local errors in $Q_{n+1}^{T} \delta_{n+1}$ and in $Q_{n+1}^{T} E_{n}\left(h_{n}\right)$ when componentwise local errors are satisfied in $y, Q$, and $R$.

Lemma 6.1. Let $\eta_{\mathrm{U}}$ and $\eta_{\mathrm{CS}}$ denote the local errors in the components of $y$, the columns of $Q$, and the rows of $R_{n}$ corresponding to the indices $\mathrm{U}$ and $\mathrm{CS}$, respectively, with $\eta_{\mathrm{U}} \geq \eta_{\mathrm{CS}}$. Then we have

$$
\left|\left(Q_{n+1}^{T} E_{n}\left(h_{n}\right)\right)_{i j}\right| \leq\left(\chi_{\{i \leq j\}}+\sum_{k=1}^{j}\left|\left(R_{n}\right)_{k j}\right|\right) \eta_{\iota}+O\left(\eta_{\mathrm{U}}^{2}\right)=:\left(\chi_{\{i \leq j\}}+\sigma_{n j}\right) \eta_{\iota}+O\left(\eta_{\mathrm{U}}^{2}\right),
$$

where $\iota:=\mathrm{U}$ for $i \in \mathrm{U}, \iota:=\mathrm{CS}$ for $i \in \mathrm{CS}$, and $\chi_{\{i \leq j\}}$ is 1 for $i \leq j$ and 0 otherwise.

Proof. The inequality (6.2) follows using (4.4) and the fact that we have $x_{n+1}-$ $\phi\left(x_{n}, h_{n}\right)=\delta_{n+1}$. The inequality (6.3) follows similarly.

Remark 6.1. Note that if we bound the local error for the $i$ th column of $Q$ by $\eta_{\iota} /\left(1+\max \left\{\left\|R_{n}\right\|_{1},\left\|x_{n+1}\right\|_{1}\right\}\right)$, then the bounds (6.2) and (6.3) in Lemma 6.1 become $2 \eta_{\iota}+O\left(\eta_{\mathrm{U}}^{2}\right)$ and $\left(\chi_{\{i \leq j\}}+1\right) \eta_{\iota}+O\left(\eta_{\mathrm{U}}^{2}\right)$, respectively, where $\iota:=\mathrm{U}$ for $i \in \mathrm{U}$, and $\iota:=\mathrm{CS}$ for $i \in \mathrm{CS}$.

Given the bound (6.3) for $Q_{n+1}^{T} E_{n}(t)$ we focus on determining componentwise bounds on $Q_{n+1}^{T}\left[D_{w_{n}} \phi\left(w_{n}, h_{n}\right)-D_{x_{n}} \phi\left(x_{n}, h_{n}\right)\right]$. Consider the linear equations

$$
Q_{n+1}^{T} \dot{W}=\left[Q_{n+1}^{T} D f\left(\phi\left(w_{n}, t\right)\right) Q_{n+1}\right] Q_{n+1}^{T} W \equiv B(t) Q_{n+1}^{T} W, W(0)=I,
$$

and

$$
Q_{n+1}^{T} \dot{X}=\left[Q_{n+1}^{T} D f\left(\phi\left(x_{n}, t\right)\right) Q_{n+1}\right] Q_{n+1}^{T} X \equiv C(t) Q_{n+1}^{T} X, X(0)=I .
$$


Let $V(t):=Q_{n+1}^{T}[W(t)-X(t)]$ and observe that $V(0)=0$. Then we have

$$
\dot{V}=B(t) V+[B(t)-C(t)]\left(Q_{n+1}^{T}\left[Y_{n}(t)+E_{n}(t)\right]\right) .
$$

DeFinition 6.1. Define $a$ and $b_{j}$ as constants that satisfy $a \geq \sup _{0 \leq t \leq h_{n}}\|B(t)\|_{\infty}$ and $b_{j} \geq \sup _{0 \leq t \leq h_{n}}\left\|\Pi_{j}\right\|_{\infty}$, where $\Pi_{j}$ denotes the $j$ th column of

$$
[B(t)-C(t)]\left(Q_{n+1}^{T}\left[Y_{n}(t)+E_{n}(t)\right]\right) .
$$

Lemma 6.2. With the assumptions of Lemma 6.1 we have

$$
\left|\left(Q_{n+1}^{T}\left[D_{w_{n}} \phi\left(w_{n}, h_{n}\right)-D_{x_{n}} \phi\left(x_{n}, h_{n}\right)\right]\right)_{i j}\right| \leq \frac{b_{j}}{a}\left(\exp \left(a h_{n}\right)-1\right) .
$$

Proof. Let $z_{j}(t)$ denote the supremum norm of the $j$ th column of $V(t)$. Then we have

$$
\frac{d}{d t} z_{j}(t) \leq a z_{j}(t)+b_{j}
$$

so by Gronwall's inequality (see [25, p. 108]) and using the fact that $z_{j}(0)=0$, we have

$$
z_{j}\left(h_{n}\right) \leq \frac{b_{j}}{a}\left(\exp \left(a h_{n}\right)-1\right)
$$

Using (6.3) and (6.8), we obtain the following bounds on the magnitude of the components of $Q_{n+1}^{T}\left[D_{w_{n}} \phi\left(w_{n}, h_{n}\right)-Y_{n}\right]$.

Corollary 6.1. With the assumptions of Lemma 6.1 we have

$$
\begin{gathered}
\left|\left(Q_{n+1}^{T}\left[D_{w_{n}} \phi\left(w_{n}, h_{n}\right)-Y_{n}\right]\right)_{i j}\right| \leq\left(\chi_{\{i \leq j\}}+\sum_{k=1}^{j}\left|\left(R_{n}\right)_{k j}\right|\right) \eta_{\iota}+O\left(\eta_{\mathrm{U}}^{2}\right) \\
+\frac{b_{j}}{a}\left(\exp \left(a h_{n}\right)-1\right)=:\left(\chi_{\{i \leq j\}}+\sigma_{n j}\right) \eta_{\iota}+\rho_{n j}+O\left(\eta_{\mathrm{U}}^{2}\right),
\end{gathered}
$$

where $\iota:=\mathrm{U}$ for $i \in \mathrm{U}$, and $\iota:=\mathrm{CS}$ for $i \in \mathrm{CS}$.

Our task now is to determine computable bounds on $a$ and $b_{j}$. The difficulty occurs because the matrix functions $B(t)$ and $C(t)$ are not explicitly known.

We assume that either $f$ satisfies a one-sided Lipschitz condition (see [25, p. 173]) so there exists a constant $L_{\mathrm{os}}$ such that

$$
\left\langle f\left(w_{n}(t)\right)-f\left(x_{n}(t)\right), w_{n}(t)-x_{n}(t)\right\rangle \leq L_{\mathrm{os}}\left\|w_{n}(t)-x_{n}(t)\right\|_{2}^{2},
$$

or $f$ is locally Lipschitz so that there exists a nonnegative constant $L_{\text {loc }}$ such that

$$
\left\|f\left(w_{n}(t)\right)-f\left(x_{n}(t)\right)\right\|_{\infty} \leq L_{\mathrm{loc}}\left\|w_{n}(t)-x_{n}(t)\right\|_{\infty} .
$$

Using Gronwall's inequality for $\Lambda=L_{\mathrm{os}}$ (and $\left.\varepsilon_{0}=\sqrt{N} \epsilon_{0}\right)$ or $\Lambda=L_{\mathrm{loc}}\left(\right.$ and $\left.\varepsilon_{0}=\epsilon_{0}\right)$, respectively, we have

$$
\left\|w_{n}(t)-x_{n}(t)\right\|_{\infty} \leq \exp (\Lambda t) \cdot \varepsilon_{0} .
$$

Let $L_{D f}$ denote the Lipschitz constant for $D f$ so that

$$
\begin{aligned}
& \left\|D f\left(w_{n}(t)\right)-D f\left(x_{n}(t)\right)\right\|_{\infty} \leq L_{D f}\left\|w_{n}(t)-x_{n}(t)\right\|_{\infty}, \\
& \left\|D f\left(x_{n}(t)\right)-D f\left(y_{n}(t)\right)\right\|_{\infty} \leq L_{D f}\left\|x_{n}(t)-y_{n}(t)\right\|_{\infty} .
\end{aligned}
$$


Thus,

$$
\begin{aligned}
\left|D f\left(w_{n}(t)\right)_{i j}-D f\left(x_{n}(t)\right)_{i j}\right| & \leq \varepsilon_{0} \exp (\Lambda t) L_{D f}=: G_{1}(t), \\
\left|D f\left(x_{n}(t)\right)_{i j}-D f\left(y_{n}(t)\right)_{i j}\right| & \leq L_{D f}|| \delta_{n+1}(t) \|_{\infty}=: G_{2}(t),
\end{aligned}
$$

where $\delta_{n+1}(t)$ is the local error at the $n$th step at time $t$ for $0 \leq t \leq h_{n}$. Then we have

$$
\begin{aligned}
\left|D f\left(w_{n}(t)\right)_{i j}\right| & \leq\left|D f\left(w_{n}(t)\right)_{i j}-D f\left(x_{n}(t)\right)_{i j}\right| \\
& +\left|D f\left(x_{n}(t)\right)_{i j}-D f\left(y_{n}(t)\right)_{i j}\right|+\left|D f\left(y_{n}(t)\right)_{i j}\right| \\
& \leq G_{1}(t)+G_{2}(t)+\left|D f\left(y_{n}(t)\right)_{i j}\right|=: H_{i j}(t) .
\end{aligned}
$$

We have the following for $a$ :

$$
a:=\sup _{0 \leq t \leq h_{n}} \max _{1 \leq i \leq N} \sum_{j=1}^{N} \sum_{k=1}^{N} \sum_{l=1}^{N}\left|\left(Q_{n+1}\right)_{k i}\right| \cdot H_{k l}(t) \cdot\left|\left(Q_{n+1}\right)_{l j}\right| .
$$

For $b_{j}$ we obtain

$$
\begin{array}{r}
b_{j}:=\sup _{0 \leq t \leq h_{n}} \sum_{i=1}^{N} \sum_{k=1}^{N} \sum_{l=1}^{N}\left|\left(Q_{n+1}\right)_{k i}\right| \cdot\left|G_{1}(t)\left(Q_{n+1}\right)_{k l}\right| \cdot\left(\left|\left(Q_{n+1}^{T} Y_{n}(t)\right)_{l j}\right|\right. \\
\left.+\left|\left(Q_{n+1}^{T} E_{n}(t)\right)_{l j}\right|\right) .
\end{array}
$$

The bounds for $a$ and $b_{j}$ given in (6.18) and (6.19), respectively, combined with Lemma 6.1 and Corollary 6.1 provide bounds on the components of $\bar{Q}^{T} F(z)$ and $\bar{Q}^{T}(D F(w)-L)$ for $\|w-z\| \leq \epsilon_{0}$ when $\theta=0$.

When $\theta>0$, we need to determine bounds on

$$
Q_{n+1}^{T}\left[D_{w_{n}} \phi\left(w_{n}, t\right)-D_{w_{n}} \phi\left(w_{n}, h_{n}\right)\right] \quad \text { and } \quad Q_{n+1}^{T}\left[f\left(\phi\left(w_{n}, t\right)\right)-f_{n+1}\right]
$$

for $\left|t-h_{n}\right| \leq \theta \epsilon$ and ||$w_{n}-x_{n} \|_{\infty} \leq \epsilon_{0}$.

Definition 6.2. Define constants such that $a_{\theta}$ satisfies $a_{\theta} \geq \sup _{\left|t-h_{n}\right| \leq \theta \epsilon}\|B(t)\|_{\infty}$, $b_{\theta, j}$ satisfies $b_{\theta, j} \geq \sup _{\left|t-h_{n}\right| \leq \theta \epsilon}|| \Lambda_{j} \|_{\infty}$, where $\Lambda_{j}$ denotes the $j$ th column of

$$
B(t) Q_{n+1}^{T} D_{w_{n}} \phi\left(w_{n}, h_{n}\right)
$$

and $c_{\theta}$ and $d$ satisfy

$$
c_{\theta} \geq \sup _{\left\{t: 0 \leq t \leq s,\left|s-h_{n}\right| \leq \theta \epsilon\right\}}\left\|B(t) Q_{n+1}^{T} f\left(\phi\left(w_{n}, h_{n}\right)\right)\right\|_{\infty}
$$

and

$$
d \geq \sup _{0 \leq t \leq h_{n}}\left\|[B(t)-C(t)] Q_{n+1}^{T} f\left(\phi\left(x_{n}, t\right)\right)\right\|_{\infty} .
$$

Lemma 6.3. With the assumptions of Lemma 6.1 we have for $\left|t-h_{n}\right| \leq \theta \epsilon$,

$$
\left|\left(Q_{n+1}^{T}\left[D_{w_{n}} \phi\left(w_{n}, t\right)-D_{w_{n}} \phi\left(w_{n}, h_{n}\right)\right]\right)_{i j}\right| \leq \frac{b_{\theta, j}}{a_{\theta}}\left(\exp \left(a_{\theta} \theta \epsilon\right)-1\right) .=: \gamma_{j, n} .
$$

Proof. First derive an expression similar to (6.6), and then the proof follows using the same technique as the proof of Lemma 6.2. 
We have

$$
\begin{aligned}
Q_{n+1}^{T}\left[f\left(\phi\left(w_{n}, t\right)\right)-f_{n+1}\right] & =Q_{n+1}^{T}\left[\left\{f\left(\phi\left(w_{n}, t\right)\right)-f\left(\phi\left(w_{n}, h_{n}\right)\right)\right\}\right. \\
& \left.+\left\{f\left(\phi\left(w_{n}, h_{n}\right)\right)-f\left(\phi\left(x_{n}, h_{n}\right)\right)\right\}+\left\{f\left(\phi\left(x_{n}, h_{n}\right)\right)-f_{n+1}\right\}\right]
\end{aligned}
$$

for $\left|t-h_{n}\right| \leq \theta \epsilon$. Using the fact that $Q_{n+1}^{T} f\left(\phi\left(w_{n}, t\right)\right)$ satisfies $(6.4)$ and $Q_{n+1}^{T} f\left(\phi\left(x_{n}, t\right)\right)$ satisfies (6.5), we may obtain bounds on the left-hand side of (6.23) in a similar fashion to those previously obtained.

Lemma 6.4. With the assumptions of Lemma 6.1 we have, for $\left|t-h_{n}\right| \leq \theta \epsilon$,

$$
\begin{aligned}
\left\|Q_{n+1}^{T}\left[f\left(\phi\left(w_{n}, t\right)\right)-f_{n+1}\right]\right\|_{\infty} & \leq \frac{c_{\theta}}{a_{\theta}}\left(\exp \left(a_{\theta} \theta \epsilon\right)-1\right)+\frac{d}{a}\left(\exp \left(a h_{n}\right)-1\right) \\
& +\left\|Q_{n+1}^{T}\left[f\left(\phi\left(x_{n}, h_{n}\right)\right)-f_{n+1}\right]\right\|_{\infty}:=\zeta_{n} .
\end{aligned}
$$

We now determine computable bounds on $a_{\theta}, b_{\theta, j}, c_{\theta}$, and $d_{\theta}$ in a similar spirit to those for $a$ and $b_{j}$. For $a_{\theta}$ we use (6.17) with the limits of the supremum changed to $\left|t-h_{n}\right| \leq \theta \epsilon$ in (6.18). To find the bound for $b_{\theta, j}$, we bound $B(t)$ as for $a_{\theta}$ and then bound $Q_{n+1}^{T} D_{w_{n}} \phi\left(w_{n}, h_{n}\right)$ using (6.3) and (6.8). We bound $c_{\theta}$ and $d$ in a fashion similar to that of $b_{\theta, j}$ and $b_{j}$, respectively, and employ the bound

$$
\left\|Q_{n+1}^{T}\left[f\left(\phi\left(x_{n}, h_{n}\right)\right)-f_{n+1}\right]\right\|_{\infty} \leq\left\|Q_{n+1}^{T}\right\|_{\infty} L_{f}\left\|\delta_{n+1}\right\|_{\infty} .
$$

Putting all of these bounds together we have the following theorem that summarizes the bounds for $\theta=0$ and $\theta>0$.

TheOREm 6.5. With the assumption of Lemma 6.1 , for $\theta=0$ the bounds on the nonzero components of $\bar{Q}^{T} F(z)$ are given by inequality (6.2) in Lemma 6.1, while the bounds on the nonzero components of $\bar{Q}^{T}(D F(w)-L)$ are given by inequality (6.11) in Corollary 6.1. Bounds on the quantities a and $b_{j}$ in Corollary 6.1 may be obtained using inequalities (6.12)-(6.19).

For $\theta>0$ the bounds for the nonzero components of $\bar{Q}^{T}(D F(w)-L)$ are given by combining the bound (6.11) in Corollary 6.1 with the bound (6.22) in Lemma 6.3 and the bound (6.24) in Lemma 6.4. In addition, bounds on $a_{\theta}, b_{\theta, j}, c_{\theta}$, and d may be obtained using the inequalities (6.12)-(6.25).

7. Implementation. The basic outline of our algorithm for solving and producing a shadowing distance estimate for an approximate solution of the IVP (2.1) is as follows.

Algorithm. Given constants $\epsilon_{0}>0$ and $\theta \geq 0$ and componentwise tolerances, $\eta_{\mathrm{U}} \geq \eta_{\mathrm{CS}}>0$ with respect to the indices $\mathrm{U}$ and CS.

1. Simultaneously approximate solutions to (4.1), (4.2), and (4.3).

2. Update the elements of the matrix $U U^{T}$, and store $y_{n}, Q_{n}, R_{n}$, and $f_{n+1}$.

3. Bound componentwise the quantities $\bar{Q}^{T} F(z)$ and $\bar{Q}^{T}(D F(w)-L)$ using Theorem 6.5.

4. Decompose the matrix $U U^{T}$ and form $\hat{Q} U^{\dagger}$ (see (5.3)).

5. Using the results from steps 3 and 4, estimate $\alpha$ in (3.1) and $\beta$ in (3.2) using (5.4).

6. If $\alpha<1$ and $\beta /(1-\alpha) \leq \epsilon_{0}$, then set the shadowing distance $\epsilon:=\beta /(1-\alpha)$. 
Implementation Details.

1. We employ ODEX [17] or RKSUITE [3] to approximate the differential equations and use a projected integration scheme to maintain orthogonality of $Q$ (see [9]) using a modified Gram-Schmidt procedure [14]. We modified the error control of ODEX to enforce componentwise error tolerances. The use of componentwise tolerances is a standard feature in RKSUITE. We bound the local error for the columns of $Q$ as described in Remark 6.1 and set $\mu_{n}=2$ and $\sigma_{n j}=1$ for all $n$ and $j$. For RKSUITE we employ the $(7,8)$ Runge-Kutta pair.

2. We use symmetric block tridiagonal storage when forming $U U^{T}$.

3. We estimate $\bar{Q}^{T} F(z)$ and $\bar{Q}^{T}(D F(w)-L)$ by sampling at the mesh points $\left\{t_{n}\right\}_{0}^{M}$ so that $\sup _{0 \leq t \leq h_{n}}$ is replaced by $\max _{t \in\left\{0, h_{n}\right\}}$ and we neglect the $O\left(\eta_{\mathrm{U}}^{2}\right)$ terms in (6.2) and (6.3).

4. We solve for some of the rows of $\hat{Q} U^{\dagger}$ and then use (5.4) to estimate $\alpha$ and $\beta$. This provides lower bounds on $\alpha$ and $\beta$ that will be sharp if the "correct" rows are used. We use the first and last block rows of $\hat{Q} U^{\dagger}$ and several picked at random to obtain an estimate on $\alpha$ without the full cost of solving for all of $U^{\dagger}$. To determine block rows of $U^{\dagger}$ we use the block tridiagonal solver TRDBLK [11].

8. Numerical results. In this section we present numerical results of our shadowing algorithm applied to several nonlinear IVPs. We obtain approximate solutions and local error estimates from the IVP solvers ODEX and RKSUITE. In the description of our numerical results we denote by " $M$ " the number of time steps taken; " $U$ " denotes the set of indices for which the local error tolerance " $\eta_{\mathrm{U}}$ " is employed, while the local error tolerance " $\eta_{\mathrm{CS}}$ " is used for the remaining indices. The value " $T$ " is the final time reached during the numerical computation; "QhUdnorm" is the maximum row sum of the rows of $\hat{Q} U^{\dagger}$ that were computed. For the numerical experiments recorded here we computed six block rows: the first two, last two, and middle two block rows. When $\theta>0$ we compute the corresponding additional rows of $\hat{Q} U^{\dagger}$. We found some variation in QhUdnorm when using other rows of $\hat{Q} U^{\dagger}$, but always within the same order of magnitude and, in fact, never greater than twice the value of QhUdnorm reported here. We recommend randomly choosing the rows of $\hat{Q} U^{\dagger}$ to be computed, but have chosen to compute these particular six block rows for definiteness. We also report on the value " $\alpha$ " in (3.1) and the value of the shadowing distance " $\epsilon$ " that was obtained. We will indicate with "-" if there was no value of $\epsilon_{0}$ for which we were able to satisfy $\alpha<1$ and $\epsilon \leq \epsilon_{0}$. All computations where performed on a Linux workstation with $512 \mathrm{MB}$ memory using a $400 \mathrm{MHz}$ processor. We found that the computation of $y, Q$, and $R$ comprised approximately 85 percent of the computation time when using ODEX and approximately 70 percent of the computation time when using RKSUITE.

To compare the numerical shadowing technique presented here with other shadowing techniques we consider two categories of techniques: (i) "BVP techniques"- these are based upon forming a right inverse by specifying boundary conditions using the stability properties of the system and then bounding the norm of a solution to this BVP; (ii) "pseudoinverse techniques" - this is the approach taken here and involves finding and bounding the norm of a particular right inverse, the pseudoinverse. In general, the BVP technique (see, e.g., [5], [7]) provide somewhat conservative values for the shadowing distance, but they are obtained rather inexpensively. The pseudoinverse techniques (see, e.g., [26] and this paper) are expensive, but sharper. The expense is due to the requirement that we find $L^{\dagger}$ explicitly so the norm of the pseudoinverse is obtained essentially exactly. By obtaining only selected rows of $L^{\dagger}$ 
TABLE 8.1

Forced $v d P\left(M=1 E+5, \mathrm{U}=\{1\}, \eta_{\mathrm{U}}=1 . E-6\right)$.

\begin{tabular}{|c|c|c|c|c|c|c|}
\hline Meth & $\eta_{\mathrm{CS}} / \eta_{\mathrm{U}}$ & $\theta$ & $T$ & QhUdnorm & $\alpha$ & $\epsilon$ \\
\hline RKSUITE & $1 . E 0$ & $0 . E 0$ & $1.56 E+4$ & $2.1 E+2$ & $1.3 E-1$ & $4.9 E-4$ \\
\hline RKSUITE & $1 . E 0$ & $1 . E-1$ & $1.56 E+4$ & $1.9 E+1$ & $1.9 E-2$ & $4.0 E-5$ \\
\hline RKSUITE & $1 . E-1$ & $1 . E-1$ & $1.23 E+4$ & $2.4 E+1$ & $1.9 E-2$ & $2.8 E-5$ \\
\hline RKSUITE & $1 . E-2$ & $1 . E-1$ & $9.80 E+3$ & $2.8 E+1$ & $1.9 E-2$ & $3.4 E-5$ \\
\hline ODEX & $1 . E 0$ & $0 . E 0$ & $2.58 E+4$ & $1.9 E+2$ & - & - \\
\hline ODEX & $1 . E-1$ & $0 . E 0$ & $2.27 E+4$ & $1.8 E+2$ & $6.0 E-1$ & $9.0 E-4$ \\
\hline ODEX & $1 . E-2$ & $0 . E 0$ & $1.50 E+4$ & $2.0 E+2$ & $3.4 E-1$ & $5.9 E-4$ \\
\hline ODEX & $1 . E 0$ & $1 . E-1$ & $2.58 E+4$ & $1.5 E+1$ & $1.0 E-1$ & $3.3 E-5$ \\
\hline ODEX & $1 . E-1$ & $1 . E-1$ & $2.27 E+4$ & $1.3 E+1$ & $8.3 E-2$ & $1.4 E-5$ \\
\hline ODEX & $1 . E-2$ & $1 . E-1$ & $1.50 E+4$ & $3.6 E+1$ & $6.3 E-2$ & $2.2 E-5$ \\
\hline
\end{tabular}

we have decreased the expense but at the cost of perhaps not having a bound on the norm of the entire pseudoinverse. Regardless of how the right inverse has been chosen and bounded, a result similar to Proposition 4.1 in [4] has been used (see [5], [7], and [26]) to determine the shadowing distance $\epsilon$. With the standard fixed point result, which takes $\alpha=1 / 2$ and has two additional norm bounds, the shadowing distance is approximately 2||$L^{\dagger} \| \delta$ where $\delta$ is a uniform bound on the norm of the local error. With the formulation and fixed point result presented here, for $\eta_{\mathrm{CS}} / \eta_{\mathrm{U}}=1$ the best possible shadowing distance is $\epsilon=$ QhUdnorm $\cdot 2 \eta_{\mathrm{U}}$, where $2 \eta_{\mathrm{U}}$ is our bound on the supremum norm of the local error tolerance (see Remark 6.1), and the results can only improve for $\eta_{\mathrm{CS}} / \eta_{\mathrm{U}}<1$.

Example 8.1. As our first example we consider a forced van der Pol oscillator

$$
\ddot{y}+\alpha\left(y^{2}-1\right) \dot{y}+y=\beta \cos (\omega t)
$$

with $\alpha=\frac{2}{5}, \beta=2 \alpha^{2}$, and $\omega=\sqrt{1-\alpha^{2}}$ and the initial conditions $(0,0)$. In our numerical experiments, we set $\epsilon_{0}=5 . E-4$ when using RKSUITE and set $\epsilon_{0}=1 . E-3$ when using ODEX.

In Table 8.1, we see a decrease by an order of magnitude in QhUdnorm for $\theta>0$ since one of the Lyapunov exponents is close to zero. In addition, there is some improvement in the shadowing distance $\epsilon$ when $\eta_{\mathrm{CS}} / \eta_{\mathrm{U}}<1$. There is a trade-off here in that there is some decrease in the average stepsize when $\eta_{\mathrm{CS}} / \eta_{\mathrm{U}}<1$, which tends to increase QhUdnorm, but in spite of this there is a decrease in $\epsilon$ especially for $\eta_{\mathrm{CS}} / \eta_{\mathrm{U}}=1 . E-1$. This is not a particularly good example for our method since both of the Lyapunov exponents are negative (we are using $\mathrm{U}=\{1\}$ ); nonetheless we do obtain some improvement with $\eta_{\mathrm{CS}} / \eta_{\mathrm{U}}<1$.

Example 8.2. The Lorenz equation is given by

$$
\left(\begin{array}{c}
\dot{x} \\
\dot{y} \\
\dot{z}
\end{array}\right)=\left(\begin{array}{c}
\sigma(y-x) \\
\rho x-x z-y \\
x y-\beta z
\end{array}\right) .
$$

We consider the parameter values $\sigma=10, \rho=28$, and $\beta=8 / 3$, and the initial condition is chosen to be $\left(x_{0}, y_{0}, z_{0}\right)=(0,1,0)$. In our numerical experiments, we set $\epsilon_{0}=5 . E-5$ when using RKSUITE and set $\epsilon_{0}=2 . E-6$ when using ODEX. In Table 8.2, observe the decrease by an order of magnitude in the shadowing distance approximation $\epsilon$ with RKSUITE when changing $\eta_{\mathrm{CS}} / \eta_{\mathrm{U}}$ from 1.E0 to 1.E-1. In general, we tend to observe smaller shadowing distances for ODEX than for RKSUITE, ostensibly 
TABLE 8.2

Lorenz equation $\left(M=1 E+5, \mathrm{U}=\{1\}, \eta_{\mathrm{U}}=1 . E-8, \theta=1 . E-2\right)$.

\begin{tabular}{|c|c|c|c|c|c|}
\hline Meth & $\eta_{\mathrm{CS}} / \eta_{\mathrm{U}}$ & $T$ & QhUdnorm & $\alpha$ & $\epsilon$ \\
\hline RKSUITE & $1 . E 0$ & $1.31 E+3$ & $1.4 E+3$ & $1.3 E-1$ & $3.3 E-5$ \\
\hline RKSUITE & $1 . E-1$ & $9.90 E+2$ & $1.9 E+3$ & $1.6 E-1$ & $4.9 E-6$ \\
\hline RKSUITE & $1 . E-2$ & $7.42 E+2$ & $2.5 E+3$ & $2.1 E-1$ & $7.3 E-6$ \\
\hline ODEX & $1 . E 0$ & $6.10 E+3$ & $2.4 E+2$ & $1.2 E-1$ & $1.9 E-6$ \\
\hline ODEX & $1 . E-1$ & $6.29 E+3$ & $2.6 E+2$ & $1.2 E-1$ & $6.7 E-7$ \\
\hline ODEX & $1 . E-2$ & $5.79 E+3$ & $2.8 E+2$ & $2.6 E-2$ & $4.3 E-7$ \\
\hline ODEX & $1 . E-3$ & $5.54 E+3$ & $3.1 E+2$ & $1.3 E-2$ & $3.3 E-7$ \\
\hline
\end{tabular}

TABLE 8.3

Lorenz equation $\left(M=4 E+5, \mathrm{U}=\{1\}, \eta_{\mathrm{U}}=1 . E-8, \eta_{\mathrm{CS}}=1 . E-9, \theta=1 . E-2\right)$.

\begin{tabular}{|c|c|c|c|c|c|}
\hline Meth & $\epsilon_{0}$ & $T$ & QhUdnorm & $\alpha$ & $\epsilon$ \\
\hline RKSUITE & $5 . E-6$ & $3.97 E+3$ & $1.4 E+3$ & $1.2 E-2$ & $3.0 E-6$ \\
\hline ODEX & $1 . E-6$ & $2.52 E+4$ & $1.0 E+2$ & $1.2 E-1$ & $6.7 E-7$ \\
\hline
\end{tabular}

TABLE 8.4

Coupled oscillators $\left(\mathrm{U}=\{1,2\}, \eta_{\mathrm{U}}=1 . E-10, \epsilon_{0}=2 . E-7\right)$.

\begin{tabular}{|c|c|c|c|c|c|c|}
\hline Meth & $\eta_{\mathrm{CS}} / \eta_{\mathrm{U}}$ & $\theta$ & $T$ & QhUdnorm & $M$ & $\epsilon$ \\
\hline RKSUITE & $1 . E 0$ & $0 . E 0$ & $1.07 E+2$ & $2.9 E+5$ & $1 E+4$ & - \\
\hline RKSUITE & $1 . E 0$ & $1 . E-2$ & $1.07 E+2$ & $9.1 E+2$ & $1 E+4$ & $1.8 E-7$ \\
\hline RKSUITE & $1 . E-1$ & $1 . E-2$ & $8.41 E+1$ & $1.2 E+3$ & $1 E+4$ & $1.1 E-7$ \\
\hline RKSUITE & $1 . E-2$ & $1 . E-2$ & $6.40 E+1$ & $1.7 E+3$ & $1 E+4$ & $1.3 E-7$ \\
\hline RKSUITE & $1 . E-1$ & $1 . E-2$ & $8.49 E+2$ & $1.2 E+3$ & $1 E+5$ & $1.1 E-7$ \\
\hline ODEX & $1 . E 0$ & $0 . E 0$ & $4.50 E+2$ & $4.1 E+5$ & $1 E+4$ & - \\
\hline ODEX & $1 . E 0$ & $1 . E-2$ & $4.50 E+2$ & $3.9 E+2$ & $1 E+4$ & $7.9 E-8$ \\
\hline ODEX & $1 . E-1$ & $1 . E-2$ & $4.29 E+2$ & $2.7 E+2$ & $1 E+4$ & $2.4 E-8$ \\
\hline ODEX & $1 . E-2$ & $1 . E-2$ & $4.05 E+2$ & $2.9 E+2$ & $1 E+4$ & $3.9 E-8$ \\
\hline ODEX & $1 . E-1$ & $1 . E-2$ & $4.25 E+3$ & $2.7 E+2$ & $1 E+5$ & $2.4 E-8$ \\
\hline
\end{tabular}

due to the larger average stepsize achieved by the higher order of the two methods, ODEX. In Table 8.3, we report on longer time simulations performed with $M=4 E+5$ time steps.

Example 8.3. The next example we consider is a ring of oscillators with an external force proportional to the position component of the limit cycle of a van der Pol oscillator. In particular, we consider the following system, similar to that considered in [10],

$$
\begin{aligned}
\ddot{y}+\alpha\left(y^{2}-1\right) \dot{y}+\omega^{2} y & =0 \\
\ddot{x}_{i}+d_{i} \dot{x}_{i}+\gamma\left[\Phi^{\prime}\left(x_{i}-x_{i-1}\right)-\Phi^{\prime}\left(x_{i+1}-x_{i}\right)\right] & =\sigma y \delta_{i 1}, \quad i=1, \ldots, \hat{N} .
\end{aligned}
$$

The function $\Phi(x)=\left(x^{2} / 2\right)+\left(x^{4} / 4\right)$ is the single well Duffing potential, $\alpha, \omega, \gamma, \sigma$ are scalar parameters, $x_{i}$ is the displacement of the $i$ th particle, $d_{i}$ is the damping coefficient, and we have periodic boundary conditions $\left(x_{0}=x_{\hat{N}}\right.$ and $\left.x_{\hat{N}+1}=x_{1}\right)$. For the experiments we set $\hat{N}=2, \alpha=1, \omega=1.82, \gamma=1, \sigma=4$, and $d_{1}=d_{2}=$ $3 . E-2$ and employ the initial conditions $\left(y, \dot{y}, x_{1}, \dot{x}_{1}, x_{2}, \dot{x}_{2}\right)=(1,0,0,0,0,0)$. In Table 8.4 we report on simulations for the forced coupled oscillators. The importance of rescaling of time is seen in the decrease in QhUdnorm as we increase $\theta$ from $0 . E 0$ to $1 . E-2$. In addition, we see the benefits of $\eta_{\mathrm{CS}} / \eta_{\mathrm{U}}<1$ especially when using ODEX, in particular for $\theta=1 . E-2$ we see a nontrivial decrease in the shadowing distance when $\eta_{\mathrm{CS}} / \eta_{\mathrm{U}}$ is changed from 1.E0 to $1 . E-1$. In Figure 1 we exhibit plots 

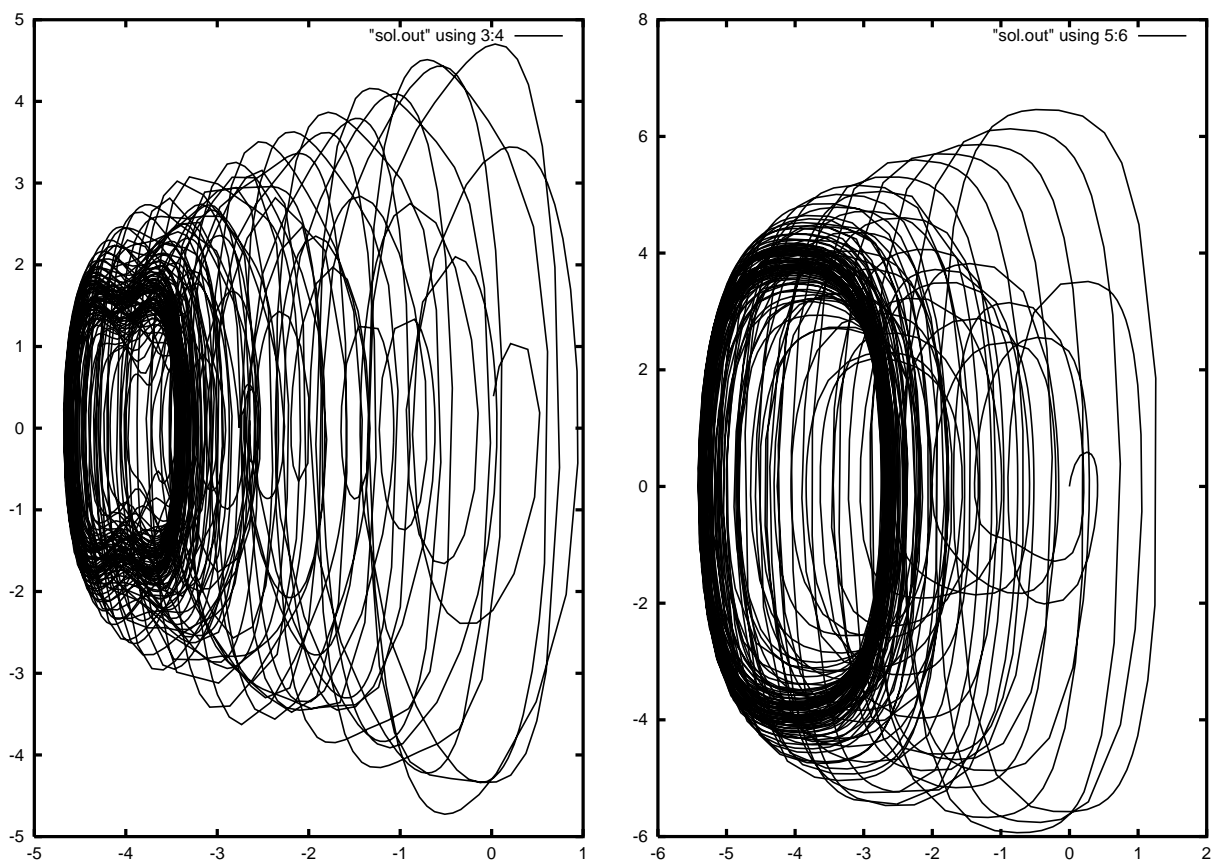

FIG. 1. Plots of solution components $\left(x_{1}, \dot{x}_{1}\right)$ and $\left(x_{2}, \dot{x}_{2}\right)$.

of the solution components $\left(x_{1}, \dot{x}_{1}\right)$ and $\left(x_{2}, \dot{x}_{2}\right)$. In Figure 2 we plot the four largest instantaneous Lyapunov exponents versus time. These instantaneous exponents are simply the diagonal elements of $\tilde{A}$ (see (4.3)), the coefficient matrix for $R$. Notice the variation of the exponents about zero which shows a lack of hyperbolicity and makes this a difficult problem for obtaining good shadowing distances. In fact, for $\theta=0$ we were not able to shadow the approximate solution.

9. Conclusions. In this paper we devised a new approach to posteriori error analysis for approximate solutions to IVP ODEs using a shadowing lemma-type approach. Using an improved fixed point theorem and a reformulation of the differential equations we were able to obtain improved shadowing results. The cost of the numerical method for providing these global error estimates is small relative to the cost of approximating the differential equations. In particular, the method is $O(M)$ in both time and memory, and we found that the cost of providing the global error estimate is a fraction of the computation time. Any improvements to the estimates in section 6 would translate into improvements in the global error estimates. The use of different local error tolerances for different components of the differential equations, made possible by the reformation of the differential and taken advantage of with the improved fixed point result, allow for improved shadowing distances without severe degradation in the average time step. In all three examples there was improvement in the shadowing distance with the use of componentwise error control. The most substantial improvement was in Example 8.2, the Lorenz equation (one positive, one zero, and one negative Lyapunov exponent), which is a good fit to our prototype example (see (5.9)-(5.11) and the discussion thereafter), while in Example 8.1 (two negative exponents) and Example 8.3 (several finite time exponents oscillating about zero) we had some improvement. 

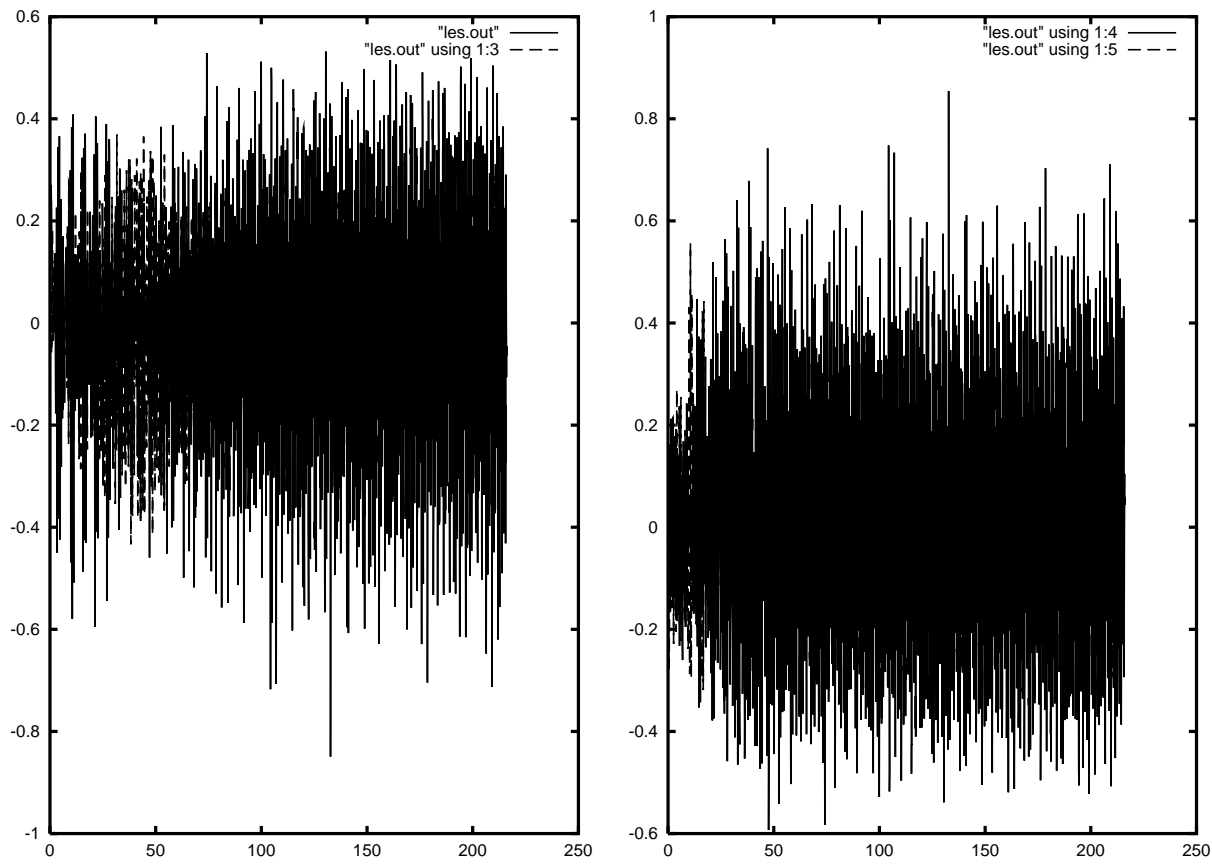

FIG. 2. Plots of first two and second two instantaneous Lyapunov exponents versus time.

Acknowledgments. We are grateful to Sebastian Reich for interesting discussions and to the referees for several helpful remarks.

\section{REFERENCES}

[1] B. Benettin And A. Giongilli, On the Hamiltonian interpolation of near the identity symplectic mappings with application to symplectic integration algorithms, J. Statist. Phys., 74 (1994), pp. 1117-1143.

[2] W.-J. BEYN, On the numerical approximation of phase portraits near stationary points, SIAM J. Numer. Anal., 24 (1987), pp. 1095-1113.

[3] R. W. Brankin, I. Gladwell, and L. F. Shampine, RKSUITE: A Suite of Runge-Kutta Codes for the Initial Value Problem for ODEs, Softreport 91-1, Mathematics Department, Southern Methodist University, Dallas, TX, 1991.

[4] S.-N. Chow, X.-B. Lin, And K. J. PALmer, A shadowing lemma with applications to semilinear parabolic equations, SIAM J. Math. Anal., 20 (1989), pp. 547-557.

[5] S. N. Chow ANd K. J. Palmer, On the numerical computation of orbits of dynamical systems: The higher dimensional case, J. Complexity, 8 (1992), pp. 398-423.

[6] B. A. Coomes, Shadowing orbits of ordinary differential equations on invariant submanifolds, Trans. Amer. Math. Soc., 349 (1997), pp. 203-216.

[7] B. A. Coomes, H. Kocak, And K. J. Palmer, Rigorous computational shadowing of orbits of ordinary differential equations, Numer. Math., 69 (1995), pp. 401-421.

[8] S. Dawson, C. Grebogi, T. Sauer, and J. A. Yorke, Obstructions to shadowing when a Lyapunov exponent fluctuates about zero, Phys. Rev. Lett., 73 (1994), pp. 1927-1930.

[9] L. DieCi AND E. S. VAN VLeCK, Computation of orthonormal factors for fundamental solution matrices, Numer. Math., 83 (1999), pp. 599-620.

[10] U. Dressler, Symmetry property of the Lyapunov spectra of a class of dissipative dynamical systems with viscous damping, Phys. Rev. A, 38-4 (1988), pp. 2103-2109.

[11] G. Fairweather, private communication, 1999.

[12] J. E. Franke and J. F. Selgrade, Hyperbolicity and chain recurrence, J. Differential Equation, 26 (1977), pp. 27-36.

[13] J. E. Franke AND J. F. Selgrade, A computer method for verification of asymptotically stable 
periodic orbits, SIAM J. Math. Anal., 10 (1979), pp. 614-628.

[14] G. H. Golub and C. F. Van Loan, Matrix Computations, The Johns Hopkins University Press, Baltimore, MD, 1989.

[15] E. HAIRER, Backward error analysis of numerical integrators and symplectic methods, Ann. Numer. Math., 1 (1994), pp. 107-132.

[16] E. HAIRER AND C. LUBICH, The life-span of backward error analysis for numerical integrators, Numer. Math., 76 (1997), pp. 441-462.

[17] E. Hairer, S. P. Norsett, And G. Wanner, Solving Ordinary Differential Equations I: Nonstiff Problems, Springer-Verlag, Berlin, 1987.

[18] S. Hammel, J. A. Yorke, And C. Grebogi, Do numerical orbits of chaotic dynamical processes represent true orbits?, J. Complexity, 3 (1987), pp. 136-145.

[19] S. Hammel, J. A. Yorke, and C. Grebogi, Numerical orbits of chaotic processes represent true orbits, Bull. Amer. Math. Soc., 19 (1988), pp. 465-470.

[20] L. V. Kantorovich and G. P. Akilov, Functional Analysis, 2nd ed., Pergamon Press, Elmsford, NY, 1982.

[21] G. Meurant, A review on the inverse of symmetric tridiagonal and block tridiagonal matrices, SIAM J. Matrix Anal. Appl., 13 (1992), pp. 707-728.

[22] K. J. PAlmer, Shadowing and Silnikov chaos, Nonlinear Anal., 27 (1996), pp. 1075-1093.

[23] S. REICH, Backward error analysis for numerical integrators, SIAM J. Numer. Anal., 36 (1998), pp. 1549-1570.

[24] T. Sauer And J. A. Yorke, Rigorous verification of trajectories for computer simulations of dynamical systems, Nonlinearity, 4 (1991), pp. 961-979.

[25] A. M. Stuart and A. R. Humphries, Dynamical Systems and Numerical Analysis, Cambridge University Press, London, 1996.

[26] E. S. VAN Vleck, Numerical shadowing near hyperbolic trajectories, SIAM J. Sci. Comput., 16 (1995), pp. 1177-1189. 